\title{
Online Survey of Clinical Practice in Patients with Schizophrenia Treated with Long-Acting Injectable Aripiprazole or Paliperidone Palmitate
}

\author{
Pedro Such ${ }^{1}$ \\ José Manuel Olivares ${ }^{2}$ \\ Lizbeth Arias ${ }^{\prime}$ \\ Mette Troels Berg' \\ Jessica Madera ${ }^{3}$ \\ 'Medical Strategy and Communication, \\ H. Lundbeck A/S, Valby, 2500, Denmark; \\ ${ }^{2}$ Department of Psychiatry, Hospital \\ Álvaro Cunqueiro, Health Research \\ Institute Galicia Sur (IISGS), CIBERSAM, \\ Vigo, Spain; ${ }^{3}$ Global Medical Affairs, \\ Otsuka Pharmaceutical Development \& \\ Commercialization, Inc, Princeton, New \\ Jersey, 08540, USA
}

Background: To obtain real-world evidence of functional improvements during atypical long-acting injectable (aLAI) therapy in recent-onset schizophrenia, an online survey was conducted to assess the impact of aripiprazole once-monthly injectable $400 \mathrm{mg}$ (AOM 400; partial $\mathrm{D}_{2}$ receptor agonist) and paliperidone palmitate once-monthly (PP1M; injectable, full $\mathrm{D}_{2}$ receptor antagonist).

Methods: Psychiatrists provided data for their 2 most recent AOM 400 patients, 2 most recent PP1M patients. Survey included 2000 patient cases (1000 AOM 400; 1000 PP1M). Eligible patients were aged 18-35 years, had been diagnosed with schizophrenia within 5 years, received AOM 400 or PP1M continuously for $\geq 6$ months according to approved labels (mean durations: 1.6 and 1.7 years with AOM 400 and PP1M, respectively). Assessments included Global Assessment of Functioning (GAF) Scale, Personal and Social Performance Scale, Positive and Negative Syndrome Scale, and Quality of Life Scale. GAF assessments were done retrospectively and also at the time of survey.

Results: Baseline mean GAF scores were 43.3 and 43.8 for AOM 400 and PP1M, respectively, indicating serious symptoms/functional impairment in both groups. Mean improvements following AOM 400 and PP1M therapy were 19.7 and 16.3 points, respectively (final scores in mild functional impairment category). Other measures assessing symptoms/functionality/quality of life demonstrated the benefits of long-term aLAI therapy.

Conclusion: Schizophrenia patients with serious functional impairment prior to current aLAI treatment showed improvements in functional outcome after AOM 400 or PP1M therapy. These results suggest functional improvements with aLAIs are apparent not only in research but also real-world settings.

Keywords: antipsychotic treatment, aripiprazole, aripiprazole once-monthly injectable, AOM, long-acting injectable, paliperidone palmitate once-monthly injectable

\section{Introduction}

The chronic relapsing-remitting course of schizophrenia necessitates continuous treatment to maintain symptom control. Studies have shown that patients who maintain symptomatic remission have improved health-related quality of life (HRQOL) and are more likely to maintain good functional status, ${ }^{1,2}$ and that poor HRQOL and lower scores on measures of functioning are associated with higher risk of initial or subsequent relapse and hospitalization. ${ }^{3,4}$ From an economic perspective, one study also showed that patients' global functioning correlated negatively with the indirect costs of schizophrenia (ie, those not directly related
Correspondence: Pedro Such

Medical Strategy and Communication

H. Lundbeck A/S, Ottiliavej 9, Valby, 2500,

Denmark

Tel +453630 I3II

Email PSU@lundbeck.com 
to psychiatric services or other medical treatment). ${ }^{5}$ Critically, it has been demonstrated that patients who relapse may not regain prerelapse functional status even after symptom remission is restored. ${ }^{6}$ From the evidence demonstrated in all these studies has come the emerging concept that assessment of patient functioning and HRQOL can provide a more nuanced and comprehensive profile of treatment efficacy compared with symptomatic relief alone. ${ }^{7,8}$

With respect to treatment strategy, the devastating, cumulative, long-term impact of relapse and the adverse influence of functional deficits, even early in the course of the disease, highlight the need for early antipsychotic therapy to establish consistent, durable symptom control in schizophrenia. ${ }^{9}$ Nonadherence that results in substantial treatment gaps is highly prevalent in patients who receive daily oral agents; ${ }^{10,11}$ thus, long-acting injectable (LAI) antipsychotic medications offer an opportunity to provide more consistent symptom control. Administration of LAIs instead of oral agents after a schizophrenia-related hospitalization has been found to improve adherence and decrease hospitalization risk; ${ }^{12}$ in addition, initiation of an LAI instead of an oral agent early in treatment (after a first schizophrenic episode) has been found to decrease the risk of subsequent hospitalization ${ }^{13}$ and improve functional outcomes. ${ }^{14,15}$ Further, a meta-analysis of mirrorimage studies comparing periods of oral antipsychotic vs LAI treatment in the same patients showed the strong superiority of LAIs in preventing hospitalization. ${ }^{16}$ Given these advantages, LAIs are being increasingly considered as a first-line option in patients with early schizophrenia. ${ }^{9,17}$

Paliperidone palmitate once-monthly (PP1M), a full $\mathrm{D}_{2}$ receptor antagonist, was approved for acute or maintenance treatment of adult schizophrenia in 2009 and 2010 in the United States and European Union, respectively. The clinical trial program consisted of 4 acute symptom control studies ${ }^{18-21}$ and 1 longer-term maintenance study. $^{22}$ The acute control studies demonstrated that PP1M was significantly more effective than placebo for improving Positive and Negative Syndrome Scale (PANSS) total symptom scores in patients with schizophrenia. Results of the maintenance study showed that PP1M was associated with a significant delay in time to first relapse and significant reduction in overall relapse rate vs placebo.

More recently, in 2013, the aLAI antipsychotic aripiprazole once-monthly injectable $400 \mathrm{mg}$ (AOM 400) was approved in the European Union for maintenance treatment of schizophrenia in adult patients stabilized with oral aripiprazole and in the United States for the treatment of schizophrenia in adults. ${ }^{23,24}$ These approvals were based on two pivotal trials that established therapeutic equivalence of AOM 400 to oral aripiprazole in relapse prevention and a statistically significant advantage over placebo in delaying time to relapse during maintenance therapy, as well as good overall tolerability. ${ }^{25,26}$ AOM 400 offers a unique mechanism of action-partial D2 agonistwhich has been associated with symptom stability and relapse prevention. ${ }^{25-28}$

In the QUAlity of LIfe with AbiliFY Maintena (QUALIFY) study (ClinicalTrials.gov NCT01795547), AOM 400 was associated with improved functional/ HRQOL outcomes relative to PP1M. ${ }^{29-32}$ Clinical trials are conducted under tightly controlled conditions, however, and the results do not always translate into realworld practice. ${ }^{9,16,33}$ Thus, it is important to confirm that improvements in functional status observed in a research setting can be replicated in typical, real-life clinical settings.

The Global Assessment of Functioning (GAF) scale, ${ }^{34}$ an instrument measuring psychological, social, and occupational functioning on a scale from 1 (lowest function) to 100 (highest function), has gained increasing acceptance as a means of assessing functionality in patients with schizophrenia, having been validated in multiple studies in this patient population. ${ }^{4,35-38}$ To obtain real-world evidence of functional improvements during aLAI therapy, a survey of psychiatrists was conducted to quantify the relative impact of AOM 400 and $\mathrm{PP} 1 \mathrm{M}$ on clinical, functional, and HRQOL outcomes, as assessed by GAF and other measures, in a variety of clinical settings. Given the importance of effective early treatment, psychiatrists were asked to provide data on relatively young, adult patients with recent-onset schizophrenia.

\section{Methods}

\section{Survey Design}

This was an online survey of psychiatrists practicing in Australia, Canada, France, Italy, Spain, and the United States. Psychiatrists surveyed had been practicing for 3-35 years, were actively treating patients $\geq 70 \%$ of their working time, were members of market research agency panels, had selected and initiated drug treatment for schizophrenia, and had seen $\geq 2$ patients with AOM 400 and $\geq 2$ 
with PP1M in the previous 3 months. Eligible psychiatrists were asked to complete clinical, functional, and HRQOL assessments based on patient record forms for the 2 most recent patients they had treated in their clinic with AOM 400 and the 2 most recent patients they had treated with PP1M as monotherapy. Participating psychiatrists were told that a pharmaceutical company was the survey sponsor but were blinded to the sponsor's identity; they were also informed that the results would be submitted for publication. Patients who met inclusion criteria for the analysis were aged 18-35 years, had been diagnosed with schizophrenia within the last 5 years, and had received AOM 400 or PP1M as monotherapy according to the approved labels for $\geq 6$ months without interruption. Data were collected for 200 patients in Australia, 280 in Canada, 360 apiece in France, Italy, and Spain, and 440 in the United States (data for a total of 2000 patients); data collection was stopped when these targets had been attained for each country. The aggregated patient cases per country were not weighted, and thus do not represent the actual size of the respective countries' population of patients diagnosed with schizophrenia, in relation to other countries.

\section{Data Collected}

All data were collected by the psychiatrists via review of patient records. The fieldwork/data collection took place between July 2, 2019 and August 19, 2019. Psychiatrist characteristics collected at the time of the survey were primary work setting, years of experience, workload, number of adult patients with schizophrenia treated with AOM 400 , PP1M, or another aLAI in the previous 3 months, and number of patients treated in the previous 3 months who met survey inclusion criteria.

Patient personal characteristics collected at the time of the survey were gender, age, living situation, employment status, and level of education. Patient clinical characteristics collected were time since schizophrenia diagnosis, psychiatric comorbidities at initiation of the current AOM 400 or PP1M therapy, and schizophrenia symptoms dominating at initiation of the respective drug. Other data collected at the time of the survey were factors relating to current use of AOM 400 or PP1M (time since initiation and current dose) and prior antipsychotic treatment (not including the respective oral version of the aLAI if it was given as a transitional agent).

Clinical outcomes assessed at the time of the survey were Clinical Global Impression (CGI)-Severity score,
CGI-Improvement score compared with the patient's condition prior to initiation of AOM 400 or PP1M, and PANSS, ${ }^{39}$ a 30 -item instrument that rates individual schizophrenia symptoms on 7-point scales ranging from 1 (absent) to 7 (extreme). For the latter, assessments were conducted for 12 of 30 items: those included in the 6-item (delusions, conceptual disorganization, hallucinatory behavior, blunted affect, passive/apathetic social withdrawal, and lack of spontaneity/flow of conversation) PANSS (PANSS-6); ${ }^{40}$ those in the 7-item (blunted affect, emotional withdrawal, poor rapport, passive/apathetic social withdrawal, lack of spontaneity/flow of conversation, motor retardation, and active social avoidance) Marder factor for Negative Symptoms; ${ }^{41}$ and 2 additional items, uncooperativeness and disturbance of volition.

Functional assessments included the following: GAF (assessed retrospectively for the condition at initiation of the current treatment and at the time of the survey), a 100point scale with scores ranging from 100 (superior functioning in a wide range of activities) to 1 (persistent danger of severely hurting self or others, persistent inability to maintain minimal personal hygiene, or serious suicidal act with clear expectation of death); ${ }^{34}$ the Personal and Social Performance scale (PSP; assessed at the time of the survey), a clinician-rated instrument that rates personal and social functioning across 4 domains (socially useful activities, personal and social relationships, self-care, and disturbing and aggressive behaviors; scale of $1=$ absent to 6 $=$ very severe). $^{42}$

Health-related quality of life outcomes (assessed at the time of the survey) were based on the Quality of Life Scale (QLS), a 21-item assessment covering 4 domains: Interpersonal Relations (8 items), Instrumental Role (4 items), Intrapsychic Foundations (7 items), and Common Objects and Activities ( 2 items). ${ }^{43}$ Ten of 21 QLS items were assessed: the 7 items included in the Intrapsychic Foundations domain (sense of purpose, motivation, curiosity, anhedonia, time utilization, empathy, and capacity for engagement and emotional interaction) and the 5 items that comprise the QLS-5 scale (social initiatives, level of accomplishment, active acquaintances, motivation, and time utilization).

\section{Statistical Analysis}

Data were collected by the psychiatrists via review of patient records. This survey was not designed to generate comparative statistics for the 2 treatment groups. All results are summarized with descriptive statistics on relevant measures 
of efficacy for patients treated with LAIs. Scores for the aggregate functional/HRQOL parameters listed above (PANSS-6, PANSS/Marder factor for Negative Symptoms, and QLS/Intrapsychic Foundations domain, QLS-5) were simple totals for the individual items.

\section{Ethics Statement}

Participating psychiatrists were told that a pharmaceutical company was the survey sponsor but were blinded to the sponsor's identity; they were also informed that the results would be submitted for publication. Because there was no interventional aspect to our survey-ie, because the survey reports retrospective patient data that were provided in a fashion that preserved patient confidentiality (only participating psychiatrists were aware of the identities of the patients; identifying information was not included in the survey reports; and the results presented here are at the group level [no individual case reports])—no institutional review board/ethics committee approval was required.

\section{Results}

\section{Psychiatrist Characteristics}

Of the 500 psychiatrists who participated in the survey, $61 \%$ primarily worked in an outpatient setting and 39\% in an inpatient setting. The psychiatrists had an average of 18.5 years of experience within the specialty and had treated an average of 130 patients with schizophrenia in the previous 3 months, of which $43 \%$ were treated with an aLAI (15\% AOM $400,18 \%$ PP1M, and 10\% another aLAI). From the pool of 500 psychiatrists, patient record forms for 1000 AOM 400and 1000 PP1M-treated patients were obtained and analyzed.

\section{Patient Personal Characteristics and Schizophrenia Status}

Personal characteristics for patients included in the survey are summarized in Table 1. Of the 2000 patients in the survey (1000 with AOM 400 and 1000 with PP1M), most were men $(67 \%)$ and mean age was 28 years, with no substantive differences between the 2 treatment groups.

There were several notable differences between the treatment cohorts. More patients in the AOM 400 vs PP1M cohort were living in individual residences with a family, partner, child, or parent (60\% vs $49 \%)$, whereas more in the PP1M cohort resided in supported-living accommodations (19\% vs $14 \%$ ) or were institutionalized (7\% vs $4 \%$ ).

With respect to employment status, $24 \%$ of the overall population was employed or doing volunteer work. More patients treated with PP1M vs AOM 400 were doing volunteer work ( $9 \%$ vs $5 \%$ ), were a nonemployed spouse/homemaker $(3 \%$ vs $1 \%)$, or were incapacitated (long-term sickness leave $[6 \%$ vs $3 \%$ ] or early retirement due to disability [6\% vs 3\%]). More patients in the AOM 400 cohort were unemployed ( $53 \%$ vs $48 \%$ ) or students (14\% vs $9 \%$ ).

Most of the overall patient population (69\%) had a high school or middle school degree as their highest level of education. More patients in the AOM 400 vs PP1M cohort had high school degrees as their highest level of education (52\% vs $45 \%$ ) and more in the PP1M cohort had no degree ( $9 \%$ vs $6 \%$ ).

Table I Baseline Characteristics for Patients Included in Survey

\begin{tabular}{|c|c|c|}
\hline & $\begin{array}{l}\text { AOM } 400 \\
(n=1000)\end{array}$ & $\begin{array}{l}\text { PPIM (n } \\
=1000)\end{array}$ \\
\hline \multicolumn{3}{|l|}{ Patient gender, n (\%) } \\
\hline Male & $659(66)$ & $687(69)$ \\
\hline Female & $340(34)$ & $309(31)$ \\
\hline Mean age, y (SD) & $28(4.7)$ & $28(4.6)$ \\
\hline \multicolumn{3}{|l|}{ Living situation, $\mathrm{n}(\%)$} \\
\hline Individual residence: Alone & $184(18)$ & $207(2 I)$ \\
\hline Individual residence: Together with & $604(60)$ & $485(49)$ \\
\hline family, partner, children, or parents & & \\
\hline Supported accommodation & $138(14)$ & $192(19)$ \\
\hline Institutionalized & $39(4)$ & $65(7)$ \\
\hline Homeless & $21(2)$ & $35(4)$ \\
\hline Other & $8(1)$ & $7(\mathrm{I})$ \\
\hline Do not know/missing information & $6(1)$ & $9(1)$ \\
\hline \multicolumn{3}{|l|}{ Employment status, n (\%) } \\
\hline Paid employment or self-employed & $175(18)$ & $162(16)$ \\
\hline Voluntary/unpaid employment & $48(5)$ & $88(9)$ \\
\hline Unemployed & $532(53)$ & $478(48)$ \\
\hline Student & $138(14)$ & $89(9)$ \\
\hline Nonemployed spouse/homemaker & $13(1)$ & $32(3)$ \\
\hline Long-term sickness leave & $30(3)$ & $60(6)$ \\
\hline Early retirement due to disability & $34(3)$ & $60(6)$ \\
\hline Other & $27(3)$ & $21(2)$ \\
\hline Do not know/missing information & $3(0)$ & $10(1)$ \\
\hline \multicolumn{3}{|l|}{ Education level, $\mathrm{n}(\%)$} \\
\hline No degree/diploma & $55(6)$ & $86(9)$ \\
\hline $\begin{array}{l}\text { Elementary/primary school (or } \\
\text { equivalent) }\end{array}$ & $85(9)$ & $97(10)$ \\
\hline Middle school (or equivalent) & $203(20)$ & $215(22)$ \\
\hline High school (or equivalent) & $519(52)$ & $446(45)$ \\
\hline $\begin{array}{l}\text { University/college degree (or } \\
\text { equivalent) }\end{array}$ & $117(12)$ & $128(13)$ \\
\hline Do not know/missing information & $21(2)$ & $28(3)$ \\
\hline
\end{tabular}

Abbreviations: AOM 400, aripiprazole once-monthly injectable $400 \mathrm{mg}$; PPIM, paliperidone palmitate once-monthly; SD, standard deviation. 
Baseline schizophrenia status data for patients included in the survey are summarized in Table 2. The mean time since diagnosis was 3.1 years overall and in each treatment cohort; however, status differed substantially between the AOM 400 and PP1M groups in several respects. In the majority of patients $(53 \%)$, mixed symptoms predominated, with a greater proportion in the PP1M vs AOM 400 cohort $(56 \%$ vs $50 \%$ ). Negative symptoms dominated in more AOM 400 vs PP1M-treated patients (15\% vs $12 \%)$. Small proportions of the overall population had obsessive-compulsive symptoms $(8 \%)$ or other psychiatric comorbidities $(7 \%$; ie, any psychiatric disease that was not listed in the survey), with greater proportions of each in the PP1M cohort.

With respect to treatment patterns, patients in the PP1M cohort had been on this therapy for a mean of 1.7 years at the time of the survey compared with 1.6 years in the AOM 400 cohort. Most AOM patients (78\%) received the $400-\mathrm{mg}$ dose, whereas $48 \%$ and $38 \%$ of PP1M patients received the $150-$ and $100-\mathrm{mg}$ doses, respectively. Approximately $69 \%$ of patients (70\% with AOM 400 and $69 \%$ with PP1M) had received a different antipsychotic therapy before initiating their current aLAI for the survey. The previous antipsychotic therapy was a secondgeneration oral therapy in $\sim 69 \%$ of patients $(73 \%$ with AOM 400 and $65 \%$ with PP1M). Five percent of PP1M patients switched from AOM 400 to PP1M and $8 \%$ of
AOM patients switched from PP1M to AOM 400 at initiation of the current treatment.

\section{Level of Functioning Global Assessment of Functioning}

At treatment initiation with AOM 400 or PP1M (assessed retrospectively at the time of the survey), mean functioning according to GAF rating in the survey sample was 43.3 for AOM 400 and 43.8 for PP1M, which falls within the range indicating serious symptoms/functional impairment (scores 41-50; Figure 1).

Following treatment with the respective aLAI, GAF rating had risen (improved) to 62.9 in the AOM 400 group vs 60.0 in the PP1M group (indicating mild symptoms). Mean improvements in GAF score were 19.7 and 16.3 points in the AOM 400 PP1M groups, respectively.

\section{Personal and Social Performance}

In both groups, the average ratings regarding deficits in selfcare, and disturbing and aggressive behaviors were in the "mild" range of the PSP scale under current therapy with the respective aLAIs (Figure 2). (A mild deficit was defined as a deficit that would be known only to someone who was very familiar with the patient.) Mean ratings for self-care were 2.4 for AOM 400, 2.6 for PP1M, and 2.5 for the total population, and those for disturbing and aggressive behaviors were 2.3, 2.5 , and 2.4 , respectively.

Table 2 Schizophrenia Status of Patients Included in Survey: Baseline Characteristics

\begin{tabular}{|c|c|c|}
\hline & AOM $400(n=1000)$ & $\operatorname{PPIM}(n=1000)$ \\
\hline Mean time since diagnosis of schizophrenia, y (SD) & $3.1(1.4)$ & $3.1(1.5)$ \\
\hline \multicolumn{3}{|l|}{ Symptoms dominating at initiation of aLAI, $\mathrm{n}$ (\%) } \\
\hline Negative & $154(15)$ & $118(12)$ \\
\hline Negative and positive & $503(50)$ & $557(56)$ \\
\hline Positive & $334(33)$ & $312(3 \mid)$ \\
\hline Do not know/missing information & $9(1)$ & $13(1)$ \\
\hline \multicolumn{3}{|l|}{ Psychiatric comorbidities at initiation of aLAl, $\mathrm{n}$ (\%) } \\
\hline Depressive symptoms & $309(31)$ & $282(28)$ \\
\hline Anxiety disorders & $339(34)$ & $312(3 \mid)$ \\
\hline Substance use disorders & $364(36)$ & $389(39)$ \\
\hline Obsessive-compulsive symptoms & $64(6)$ & $90(9)$ \\
\hline Sleep disorders & $237(24)$ & $249(25)$ \\
\hline Other psychiatric comorbidities & $53(5)$ & $80(8)$ \\
\hline None & $175(18)$ & $175(18)$ \\
\hline Do not know/missing information & $10(\mathrm{I})$ & $13(1)$ \\
\hline
\end{tabular}

Abbreviations: aLAl, atypical long-acting injectable; AOM 400, aripiprazole once-monthly injectable $400 \mathrm{mg}$; PPIM, paliperidone palmitate once-monthly; SD, standard deviation. 


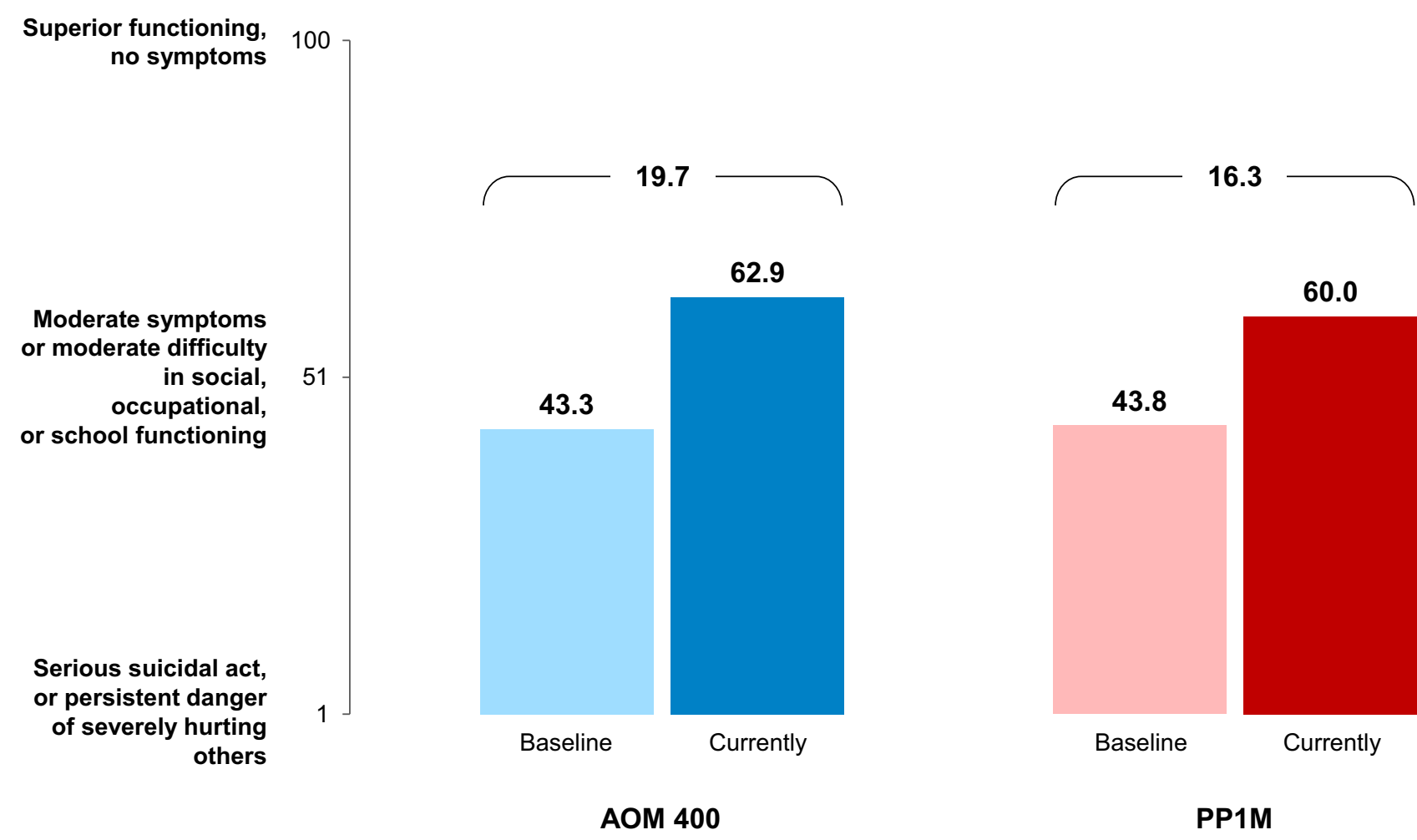

Figure I Mean Global Assessment of Functioning Scores at Baseline and on Current Atypical Long-Acting Injectable Treatment. AOM 400: aripiprazole once-monthly injectable 400 mg; PPIM: once-monthly paliperidone palmitate once-monthly. Higher scores indicate greater functionality (I-I00 scale). Numbers over brackets indicate difference between baseline and current condition. Mean durations of treatment: I.6 and I.7 years for AOM 400 and PPIM, respectively.

Deficits in self-care

(self-care includes personal hygiene, personal appearance, dressing)

Disturbing and aggressive behaviors

Deficits in personal and social relationships (relationships include those with a partner [for persons with a partner] or relatives and social relationships)

Deficits in socially useful activities,

including work or academic study (socially useful activities include cooperation with household tasks, voluntary work, "useful" hobbies such as gardening)
AOM $400 \square$ PP1M All Patients

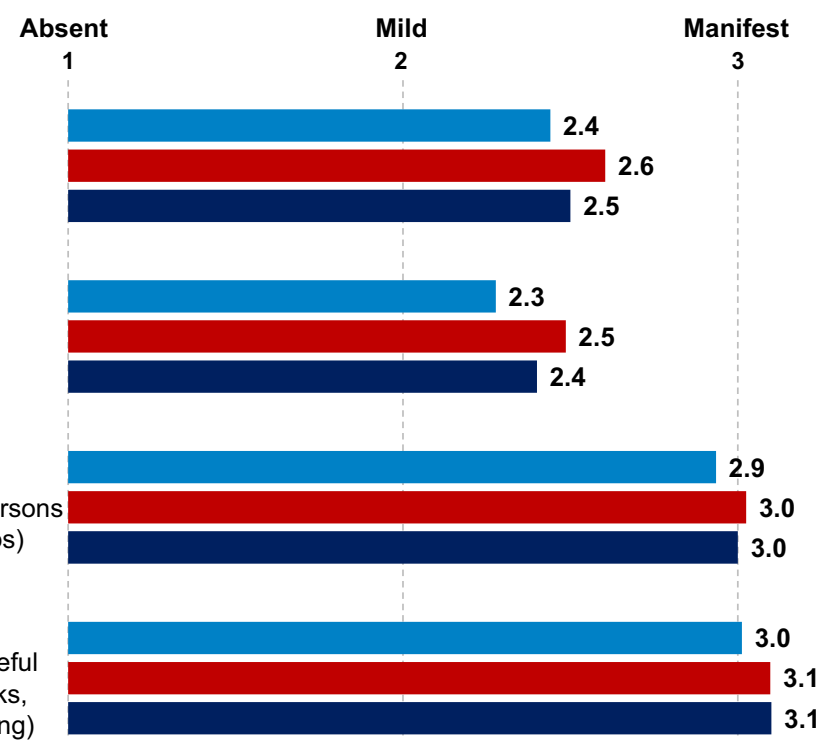

Marked

Figure 2 Personal and Social Performance Results on Current Atypical Long-Acting Injectable Treatment. AOM 400: aripiprazole once-monthly injectable 400 mg; PPIM: once-monthly paliperidone palmitate once-monthly. Lower scores indicate less severe symptoms (scale of I [absent] to 6 [very severe]). Mean durations of treatment: I.6 and I.7 years for AOM 400 and PPIM, respectively. 
Deficits in personal and social relationships were very similar for the two treatment groups: in the lower boundary of the "manifest" range in the PP1M cohort (mean 3.0) and in the upper boundary of the "mild" range in the AOM 400 cohort (mean 2.9), with an overall mean of 3.0 in the total population (Figure 2). (A manifest deficit would be clearly noticeable by everyone, but not interfere substantially with patients' ability to perform their role in that domain.)

Deficits in socially useful activities (including work or academic study) were in the manifest range in both treatment cohorts (means of 3.0, 3.1, and 3.1 for AOM 400, PP1M, and total population, respectively; Figure 2).

\section{Quality of Life Scale}

Mean ratings for the aggregate Intrapsychic Foundations domain score were 22.1 and 21.4 for AOM 400 and PP1M, respectively; corresponding mean ratings for QLS-5 were 15.5 and 15.0 (Figure 3).

\section{Clinical Evaluation \\ Clinical Global Impression of Severity and Improvement}

Based on CGI-Severity ratings obtained at the time of the survey, the majority of patients were considered mildly ill $(24 \%)$ or moderately ill $(34 \%)$ while on their aLAI, with

\section{AOM $400 \square$ PP1M}
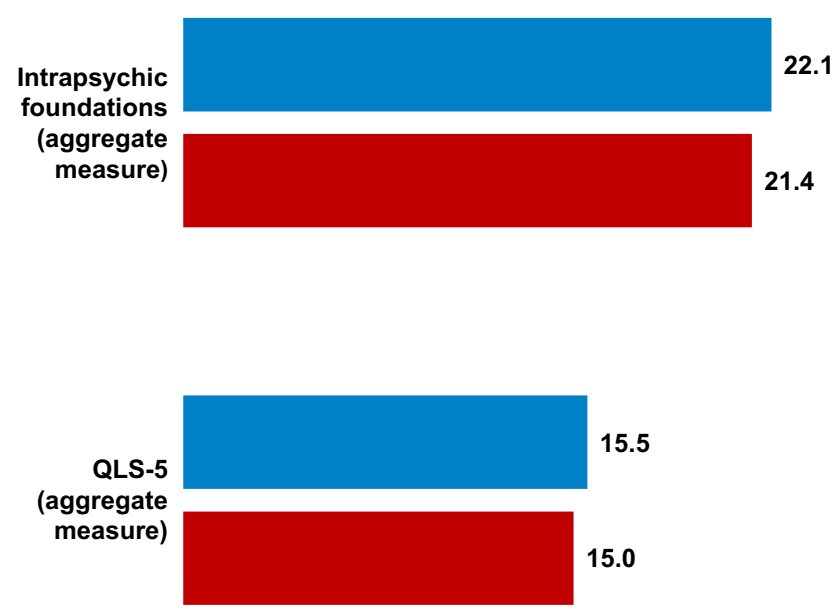

Figure 3 Aggregate Quality of Life Scale (QLS) Results on Current Atypical LongActing Injectable Treatment. AOM 400: aripiprazole once-monthly injectable 400 mg; PPIM: once-monthly paliperidone palmitate once-monthly. Higher scores indicate higher functionality/health-related quality of life (0 [worst] to 6 [best]). QLS-5 score is sum of 5 items (social initiatives, level of accomplishment, active acquaintances, motivation, and time utilization); Intrapsychic Foundations score is sum of 7 items (sense of purpose, motivation, curiosity, anhedonia, time utilization, empathy, and capacity for engagement and emotional interaction). Mean durations of treatment: 1.6 and 1.7 years for AOM 400 and PPIM, respectively.
Table 3 CGI-S Scale Score at Time of Survey

\begin{tabular}{|l|c|c|}
\hline & $\begin{array}{c}\text { AOM } 400 \text { (n = } \\
\text { I000) }\end{array}$ & $\begin{array}{c}\text { PP IM (n = } \\
\text { I000) }\end{array}$ \\
\hline Current CGI-S Scale score, & & \\
$\mathrm{n}$ (\%) & $35(4)$ & $38(4)$ \\
$\quad$ Normal, not at all ill (I) & $146(15)$ & $143(14)$ \\
Borderline mentally ill (2) & $232(23)$ & $240(24)$ \\
Mildly ill (3) & $359(36)$ & $325(33)$ \\
Moderately ill (4) & $179(18)$ & $181(18)$ \\
Markedly ill (5) & $45(5)$ & $60(6)$ \\
Severely ill (6) & $4(0)$ & $10(1)$ \\
Among the most extremely ill & & \\
patients (7) & $0(0)$ & $3(0)$ \\
Not assessed & $3.7(1.2)$ & $3.7(1.3)$ \\
Mean score (SD) &
\end{tabular}

Abbreviations: AOM 400, aripiprazole once-monthly injectable $400 \mathrm{mg}$; CGl, Clinical Global Impression-Severity; PPIM, paliperidone palmitate once-monthly.

no substantive differences in the number of AOM 400 and PP1M patients falling into the 2 respective categories (Table 3). Based on CGI-Improvement scores, most patients were very much improved or much improved (71\%) on AOM 400 or PP1M, with more on AOM 400 being very much or much improved vs PP1M (75\% vs $67 \%$; Figure 4).

\section{Positive and Negative Syndrome Scale}

Mean ratings on the aggregate PANSS items assessed (PANSS-6 and Marder factor for Negative Symptoms) were in the minimal-mild range in both the AOM 400 and PP1M cohorts (Figure 5). Mean scores for PANSS-6 were 16.4 and 17.6 for AOM 400 and PP1M, respectively; corresponding mean scores for the Marder Factor for Negative Symptoms were 20.1 and 21.3.

\section{Discussion}

Evaluating effects of treatment on functional/HRQOL outcomes requires more than a simple assessment of symptom control, as substantial proportions of patients with schizophrenia considered to be in symptomatic remission continue to suffer from functional deficits in social relations, work, and daily life activities. ${ }^{44} \mathrm{~A}$ direct comparison of functional/HRQOL and clinical outcomes with AOM 400 vs PP1M was undertaken in the QUALIFY study, in which AOM 400 was associated with significantly greater improvements vs PP1M in QLS, PSP, and CGIImprovement scores. ${ }^{29-31}$ To gather information on aLAI treatment impact in clinical practice, the present analysis surveyed psychiatrists to obtain data from real-world 


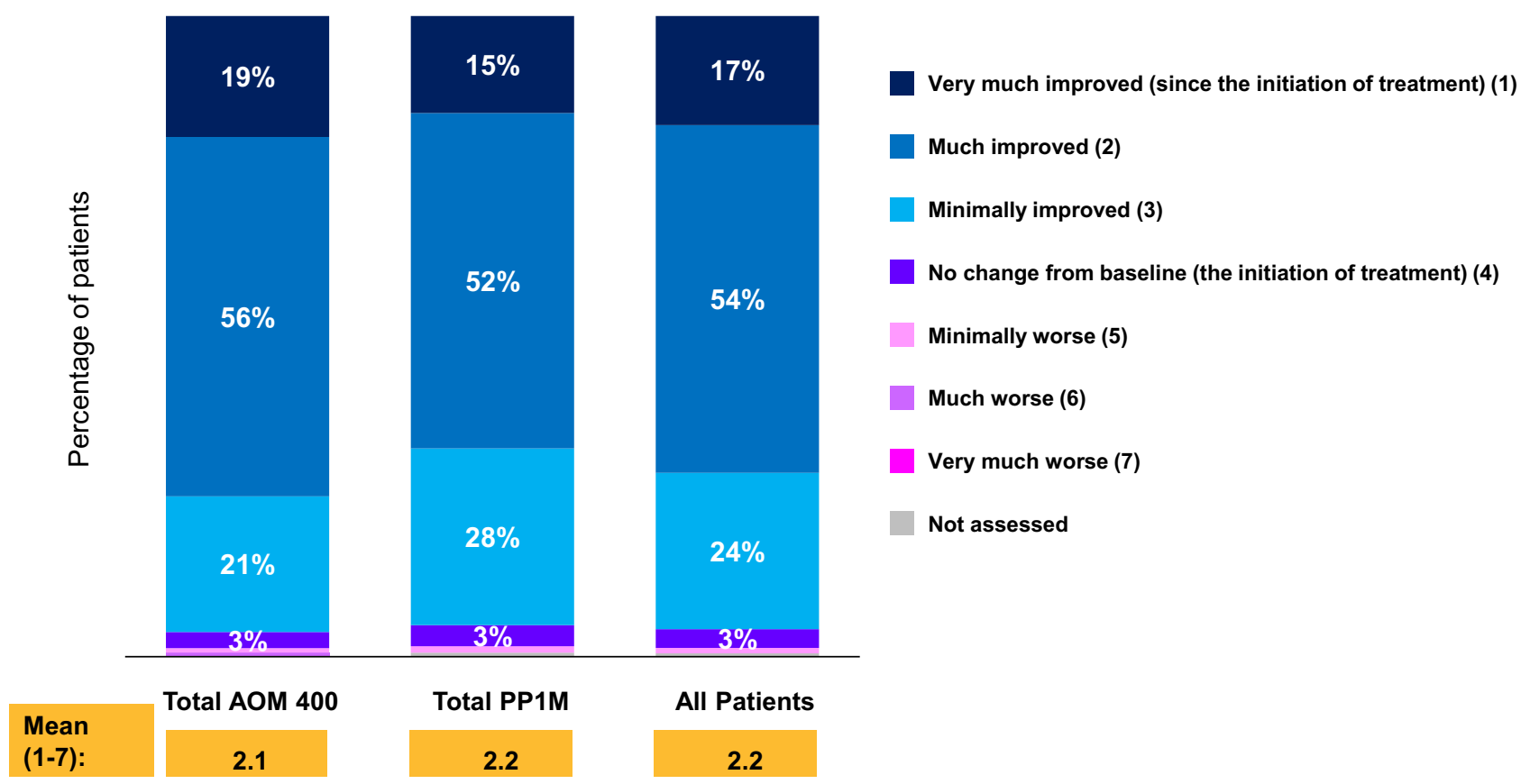

Figure 4 Clinical Global Impression-Improvement Results on Current Atypical Long-Acting Injectable Treatment. AOM 400: aripiprazole once-monthly injectable 400 mg; PPIM: once-monthly paliperidone palmitate once-monthly. Assignment to categories was based on psychiatrist's assessment of patient's condition. Mean durations of treatment: 1.6 and 1.7 years for AOM 400 and PPIM, respectively.

clinical settings regarding clinical and functional status and HRQOL in patients treated continuously with AOM 400 or PP1M for $\geq 6$ months (mean treatment durations: 1.6 and 1.7 years for AOM 400 and PP1M, respectively).

Global Assessment of Functioning was included to provide an overall snapshot of patients' social and occupational functioning. Patients in the overall cohort generally had mean GAF scores that fell within the range indicating serious symptoms/functional impairment prior to aLAI therapy. On aLAI treatment, GAF ratings improved in both cohorts, indicating mild symptoms for the AOM 400 group and borderline mild/moderate symptoms for PP1M. These findings are consistent with those of the QUALIFY study, ${ }^{29-31}$ which showed functional and clinical benefits of aLAI treatment with AOM 400 and PP1M.

The PSP was assessed at the time of the survey to characterize patients' levels of psychosocial functioning, a core feature of schizophrenia. On average, PSP scores in both treatment cohorts were in the mild or manifest range following treatment. Ratings of self-care and disturbing behaviors were mild in both cohorts; a mild deficit would only be known to someone very familiar with the patient. Deficits in socially useful activities in both cohorts were on average rated as manifest, meaning they were clearly noticeable by everyone, but did not interfere substantially with a patient's ability to perform his or her role in that domain. On average, deficits in personal and social relationships were mild in the AOM 400 cohort and slightly higher (at the low end of the manifest range) in the PP1M group, but there was no substantive difference between the 2 treatments for this domain.

The QLS was assessed at the time of the survey to gauge intrapsychic, social, and negative symptoms that contribute to deficits in functioning in schizophrenia. For the 7-item Intrapsychic Foundations domain, total scores reflected moderate functioning in both treatment groups. ${ }^{45}$

With respect to clinical outcome measures, mean ratings for the aggregate PANSS items assessed at the time of the survey (PANSS-6 and Marder factor for Negative Symptoms) fell within the minimal-mild range in both cohorts. Based on CGI-Severity ratings obtained at the time of the survey, most patients were mildly or moderately ill, with no substantive differences in disease severity between the AOM 400 and PP1M cohorts. The CGIImprovement scores at the time of the survey showed that most patients were very much improved or much improved on AOM 400 or PP1M, with more AOM 400 patients being very much or much improved.

Overall, results from this analysis are consistent with those from the QUALIFY study, ${ }^{29-31}$ which demonstrated the functional and clinical benefits of aLAI treatment with AOM 400 and PP1M, and suggest that such benefits may 
AOM 400
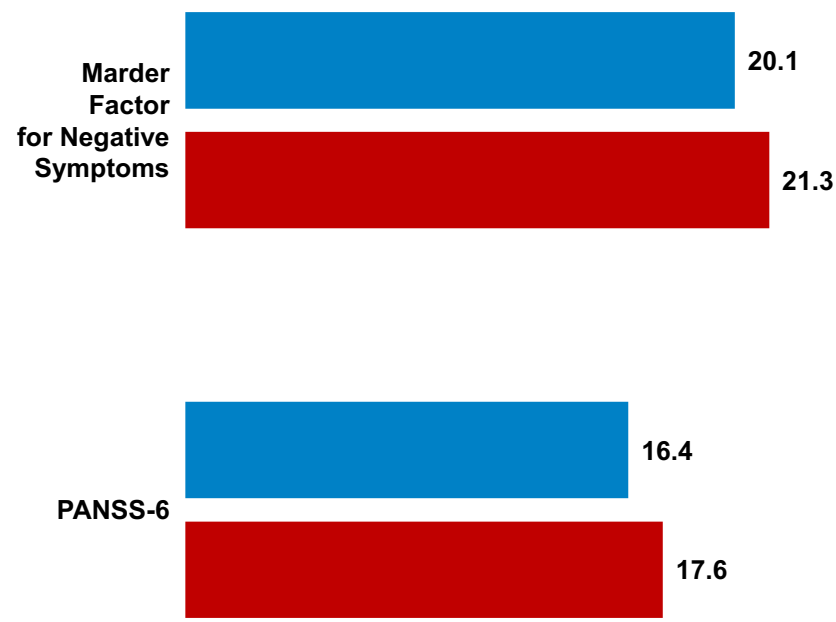

Figure 5 Positive and Negative Syndrome Scale (PANSS) Results on Current Atypical Long-Acting Injectable Treatment. AOM 400: aripiprazole once-monthly injectable $400 \mathrm{mg}$; PPIM: once-monthly paliperidone palmitate once-monthly. Mean durations of treatment: 1.6 and I.7 years for AOM 400 and PPIM, respectively Lower scores indicate less severe symptoms (7-point scale: I [absent]) to 7 [extreme]). PANSS-6 scale is sum of 6 items: delusions, conceptual disorganization, hallucinatory behavior, blunted affect, passive/apathetic social withdrawal, and lack of spontaneity/flow of conversation; PANSS Marder factor for negative symptoms is sum of 7 items (blunted affect, emotional withdrawal, poor rapport, passive/apathetic social withdrawal, lack of spontaneity/flow of conversation, motor retardation, and active social avoidance).

also be obtained in actual clinical practice. In general, the data support the feasibility of translating the results of well-controlled clinical trials into simplified measures that are applicable in real-world settings.

There are several limitations to this survey, including the use of retrospective records (patient records) to complete patient assessments and the lack of control for selection bias, as included patients had continued aLAI treatment for at least 1.6 years on average and were thus representative of a small minority of patients in clinical practice. As noted previously, the aggregated patient cases per country were not weighted, and thus did not represent the actual size of the respective countries' population of patients diagnosed with schizophrenia, in relation to other countries. The psychiatrists invited to participate in this survey were recruited from market research agency panels; as a result, there was an element of self-recruitment involved in the process, as the psychiatrists had the choice to opt in (ie, respond to the invitation) or opt out. In addition, some of the scales used in this survey (eg, GAF, PSP, QLS, and PANSS) are not typically used in clinical practice; some (eg, QLS and PANSS) were not assessed in their entirety, but rather limited to those items of the scale that were particularly relevant for the research topic at hand; and all were only assessed at the time of the survey (in the case of GAF, with an additional retrospective assessment of the patient's condition at initiation of the current treatment), so no definitive conclusions could be drawn regarding treatment effect. A further limitation in drawing comparisons between the treatments is that, although the AOM 400 and PP1M groups had similar GAF scores at initiation of aLAI therapy, the two groups might not have had comparable severity of disease at the initiation of oral treatment. It should also be noted that there were substantive disparities between the treatment groups with respect to several baseline personal characteristics and aspects of schizophrenia status, including obsessive-compulsive symptoms, which were more prevalent in the PP1M cohort and which have been shown to impact functionality, ${ }^{46}$ and other psychiatric comorbidities, a category that comprised any psychiatric disease not listed in the survey. As only descriptive statistics were used in this survey, we were not able to model for factors, such as these baseline imbalances, that could have accounted for reported differences between treatment groups.

In conclusion, patients with schizophrenia who had serious functional impairment prior to their current aLAI treatment showed improvements in functional outcome, as assessed by GAF, after 1.6 years of treatment with AOM 400 (a partial $\mathrm{D}_{2}$ agonist) or 1.7 years of treatment with PP1M (a full $\mathrm{D}_{2}$ antagonist). Other measures assessing clinical status, functionality, and quality of life (PSP, QLS, CGI-Improvement and -Severity, and PANSS) also demonstrated the benefits of long-term aLAI therapy. These results confirm that clinical and functional improvements reported with aLAIs are not only apparent in a research setting but also translate into improvements in a real-world setting.

For patients who are focused on functionality (eg, the ability to live independently, to study, to work, to have meaningful relationships, etc), rather than their symptoms, clinical practitioners should be prepared to help them achieve these goals. In this regard, naturalistic studies that assess pragmatic real-life variables may have greater positive impact on treatment practices and patients' lives than classical clinical trials, and should, therefore, be encouraged.

\section{Abbreviations}

aLAI, atypical long-acting injectable (LAI); AOM 400, aripiprazole once-monthly injectable $400 \mathrm{mg}$; CGI, Clinical Global Impression; GAF, Global Assessment of 
Functioning; HRQOL, health-related quality of life; PANSS, Positive and Negative Syndrome Scale; PP1M, paliperidone palmitate once-monthly; PANSS-6, 6-item PANSS; PSP, Personal and Social Performance scale; QLS, Quality of Life Scale; QLS-5, 5-item QLS.

\section{Role of the Funding Source}

The survey was administered by Ipsos $\mathrm{GmbH}$, Hamburg, Germany; the research survey and analysis was funded by H. Lundbeck A/S, Valby, Denmark, and Otsuka Pharmaceutical Development \& Commercialization, Inc., Princeton, NJ, USA. This manuscript was supported by Otsuka Pharmaceutical Development \& Commercialization, Inc., Princeton, NJ, USA. Editorial support, funded by Otsuka Pharmaceutical Development \& Commercialization, Inc., was provided by BioScience Communications, New York, New York, USA.

\section{Acknowledgments}

The authors wish to thank the psychiatrists who participated in the survey.

\section{Author Contributions}

All authors contributed to data analysis and drafting of the article. All authors reviewed and gave approval of all versions of the article up to and including the one used for journal submission, and they agreed on the journal to which the manuscript was submitted. All authors agree to take responsibility and be accountable for the contents of the article.

\section{Disclosure}

PS, LA, and MTB: employees of Lundbeck, JMO: paid positions, honoraria, and/or advisory boards with/received from Angelini, AstraZeneca, Bristol-Myers, Casen Recordati, Esteve, GlaxoSmithKline, Janssen, Lilly, Lundbeck, Novartis, Otsuka, Pfizer, Sanofi, and grants from National Institute of Health Carlos III, Galician Innovation Agency, and the Spanish National Plan on Drugs, JM: employee of Otsuka. The authors report no other conflicts of interest in this work.

\section{References}

1. Boden R, Sundstrom J, Lindstrom E, Lindstrom L. Association between symptomatic remission and functional outcome in first-episode schizophrenia. Schizophr Res. 2009;107(2-3):232-237. doi:10.1016/j.schres.2008.10.004
2. Karow A, Wittmann L, Schöttle D, Schäfer I, Lambert M. The assessment of quality of life in clinical practice in patients with schizophrenia. Dialogues Clin Neurosci. 2014;16(2):185-195.

3. Chen EY, Hui CL, Lam MM, et al. Maintenance treatment with quetiapine versus discontinuation after one year of treatment in patients with remitted first episode psychosis: randomised controlled trial. BMJ. 2010;341:c4024. doi:10.1136/bmj.c4024

4. Köhler O, Horsdal HT, Baandrup L, Mors O, Gasse C. Association between Global Assessment of functioning, severity, and prognosis in first-time schizophrenia. Clin Epidemiol. 2016;8:323-332. doi:10.2147/CLEP.S109036

5. Lee IH, Chen PS, Yang YK, et al. The functionality and economic costs of outpatients with schizophrenia in Taiwan. Psychiatry Res. 2008;158(3):306-315. doi:10.1016/j.psychres.2006.10.004

6. Emsley R, Chiliza B, Asmal L, Harvey BH. The nature of relapse in schizophrenia. BMC Psychiatry. 2013;13:50. doi:10.1186/1471244X-13-50

7. Kwon JS, Jung-Seok C. Social functioning and quality of life as measures of effectiveness in the treatment of schizophrenia. World Psychiatry. 2009;8(1):35-36. doi:10.1002/j.2051-5545.2009.tb00207.x

8. Peuskens J, Gorwood P. How are we assessing functioning in schizophrenia? A need for a consensus approach. Eur Psychiatry. 2012;27 (6):391-395. doi:10.1016/j.eurpsy.2011.02.013

9. Stahl SM. Long-acting injectable antipsychotics: shall the last be first? CNS Spectr. 2014;19(1):3-5. doi:10.1017/ S1092852913001016

10. Ascher-Svanum H, Faries DE, Zhu B, Ernst FR, Swartz MS, Swanson JW. Medication adherence and long-term functional outcomes in the treatment of schizophrenia in usual care. $J$ Clin Psychiatry. 2006;67(3):453-460. doi:10.4088/jcp.v67n0317

11. Velligan DI, Weiden PJ, Sajatovic M, et al. Expert consensus panel on adherence problems in serious and persistent mental illness. The expert guideline series: adherence problems in patients with serious and persistent mental illness. J Clin Psychiatry. 2009;70(Suppl 4):1-46.

12. Marcus SC, Zummo J, Pettit AR, Stoddard J, Doshi JA. Antipsychotic adherence and rehospitalization in schizophrenia patients receiving oral versus long-acting injectable antipsychotics following hospital discharge. J Manag Care Spec Pharm. 2015;21 (9):754-768. doi:10.18553/jmcp.2015.21.9.754

13. Tiihonen J, Haukka J, Taylor M, Haddad PM, Patel MX, Korhonen P. A nationwide cohort study of oral and depot antipsychotics after first hospitalization for schizophrenia. Am J Psychiatry. 2011;168 (6):603-609. doi:10.1176/appi.ajp.2011.10081224

14. Kim B, Lee SH, Choi TK, et al. Effectiveness of risperidone long-acting injection in first-episode schizophrenia: in naturalistic setting. Prog Neuropsychopharmacol Biol Psychiatry. 2008;32 (5):1231-5. doi:10.1016/j.pnpbp.2008.03.012

15. Heres S, Lambert M, Vauth R. Treatment of early episode in patients with schizophrenia: the role of long acting antipsychotics. Eur Psychiatry. 2014;29(Suppl 2):1409-1413. doi:10.1016/S09249338(14)70001-X

16. Kishimoto T, Nitta M, Borenstein M, Kane JM, Correll CU. Longacting injectable versus oral antipsychotics in schizophrenia: a systematic review and meta-analysis of mirror-image studies. J Clin Psychiatry. 2013;74(10):957-965. doi:10.4088/JCP.13r08440

17. Llorca PM, Abbar M, Courtet P, Guillaume S, Lancrenon S, Samalin L. Guidelines for the use and management of long-acting injectable antipsychotics in serious mental illness. BMC Psychiatry. 2013;13:340. doi:10.1186/1471-244X-13-340

18. Kramer M, Litman R, Hough D, et al. Paliperidone palmitate, a potential long-acting treatment for patients with schizophrenia. Results of a randomized, double-blind, placebo-controlled efficacy and safety study. Int J Neuropsychopharmacol. 2010;13(5):635-647. doi:10.1017/S1461145709990988 
19. Nasrallah HA, Gopal S, Gassmann-Mayer C, et al. A controlled, evidence-based trial of paliperidone palmitate, a long-acting injectable antipsychotic, in schizophrenia. Neuropsychopharmacology. 2010;35(10):2072-2082. doi:10.1038/npp.2010.79

20. Gopal S, Hough DW, Xu H, et al. Efficacy and safety of paliperidone palmitate in adult patients with acutely symptomatic schizophrenia: a randomized, double-blind, placebo-controlled, dose-response study. Int Clin Psychopharmacol. 2010;25(5):247-256. doi:10.1097/ YIC.0b013e32833948fa

21. Pandina GJ, Lindenmayer JP, Lull J, et al. A randomized, placebo controlled study to assess the efficacy and safety of 3 doses of paliperidone palmitate in adults with acutely exacerbated schizophrenia. J Clin Psychopharmacol. 2010;30(3):235-244. doi:10.1097/JCP.0b013e3181dd3103

22. Hough D, Gopal S, Vijapurkar U, Lim P, Morozova M, Eerdekens M. Paliperidone palmitate maintenance treatment in delaying the time-to relapse in patients with schizophrenia: a randomized, double-blind, placebo-controlled study. Schizophr Res. 2010;116(2-3):107-117. doi:10.1016/j.schres.2009.10.026

23. Abilify Maintena [package insert]. Tokyo, Japan: Otsuka Pharmaceutical Co., Ltd; revised; Jan 2020.

24. Abilify Maintena (summary of product characteristics). Amsterdam, Netherlands: Otsuka Pharmaceutical Netherlands B.V; Renewed 27 Aug 2018.

25. Kane J, Sanchez R, Perry P, et al. Aripiprazole intramuscular depot as maintenance treatment in patients with schizophrenia: a 52-week multicenter, randomized, double-blind, placebo-controlled study. J Clin Psychiatry. 2012;73(5):617-624. doi:10.4088/JCP.11m07530

26. Fleischhacker WW, Sanchez R, Perry PPM, et al. Aripiprazole once-monthly for treatment of schizophrenia: a double-blind, randomised, non-inferiority study. Br J Psychiatry. 2014;205(2):135-144. doi:10.1192/bjp.bp.113.134213

27. Peters-Strickland T, Baker RA, Such P, Zhang P, Madera JJ. The effect of aripiprazole once-monthly on personal and social functioning: post hoc analyses of acute and long-term studies. Neuropsychiatr Dis Treatment. 2019;15:1659-1669. doi:10.2147/NDT.S198241

28. Madera JJ, Such P, Zhao C, Baker RA. Symptomatic stability with aripiprazole once-monthly: efficacy analyses from acute and long-term studies. Neuropsychiatr Dis Treat. 2019;15:1593-1604. doi:10.2147/NDT.S198786

29. Naber D, Hansen K, Forray C, et al. Qualify: a randomized head-tohead study of aripiprazole once-monthly and paliperidone palmitate in the treatment of schizophrenia. Schizophrenia Res. 2015;168(1-2):498-504. doi:10.1016/j.schres.2015.07.007

30. Potkin SG, Loze J-Y, Forray C, et al. Multidimensional assessment of functional outcomes in schizophrenia: results from QUALIFY, a head-to-head trial of aripiprazole once-monthly and paliperidone palmitate. Int $J$ Neuropsychopharmacol. 2017;20(1):40-49. doi:10.1093/ijnp/pyw093

31. Potkin SG, Loze J-Y, Forray C, et al. Relationship between response to aripiprazole once-monthly and paliperidone palmitate on work readiness and functioning in schizophrenia: a post-hoc analysis of the QUALIFY study. PLoS One. 2017;12(8):e0183475. doi:10.1371/ journal.pone.0183475

32. Potkin SG, Loze J-Y, Forray C, et al. Reduced sexual dysfunction with aripiprazole once-monthly versus paliperidone palmitate: results from QUALIFY. Int Clin Psychopharmacol. 2017;32(3):147-154. doi:10.1097/YIC.0000000000000168
33. Kishimoto T, Robenzdeh A, Leucht C, et al. Long-acting injectable vs oral antipsychotics for relapse prevention in schizophrenia: a meta-analysis of randomized trials. Schizophr Bull. 2014;40 (1):192-213. doi: $10.1093 /$ schbul/sbs 150

34. Bodlund O, Kullgren G, Ekselius L, Lindstrom E, Knorring L. Axis V-global assessment of functioning scale. Acta Psychiatr Scand. 1994;90(5):342-347. doi:10.1111/j.1600-0447.1994.tb01604.x

35. Jones SH, Thornicroft G, Coffey M, Dunn G. A brief mental health outcome scale-reliability and validity of the Global Assessment of Functioning (GAF). $\quad B r \quad J \quad$ Psychiatry. 1995;166(5):654-659. doi:10.1192/bjp.166.5.654

36. Startup M, Jackson MC, Bendix S. The concurrent validity of the global assessment of functioning (GAF). Br J Clin Psychol. 2002;41 (Pt 4):417-422. doi:10.1348/014466502760387533

37. Samara MT, Engel RR, Millier A, Kandenwein J, Toumi M, Leucht S. Equipercentile linking of scales measuring functioning and symptoms: examining the GAF, SOFAS, CGI-S, and PANSS. Eur Neuropsychopharmacol. 2014;24(11):1767-1772. doi:10.1016/j. euroneuro.2014.08.009

38. Suzuki T, Uchida H, Sakurai H, et al. Relationships between global assessment of functioning and other rating scales in clinical trials for schizophrenia. Psychiatry Res. 2015;227(2-3):265-269. doi:10.1016/ j.psychres.2015.02.024

39. Kay SR, Fiszbein A, Opler LA. The Positive and Negative Syndrome Scale (PANSS) for schizophrenia. Schizophr Bull. 1987;13 (2):261-276. doi:10.1093/schbul/13.2.261

40. Østergaard SD, Lemming OM, Mors O, Correll CU, Bech P. PANSS6: a brief rating scale for the measurement of severity in schizophrenia. Acta Psychiatr Scand. 2016;133(6):436-444. doi:10.1111/acps. 12526

41. Marder SR, Davis JM, Chouinard G. The effects of risperidone on the five dimensions of schizophrenia derived by factor analysis: combined results of the North American trials. J Clin Psychiatry. 1997;58 (12):538-546. doi:10.4088/jcp.v58n1205

42. Morosini PL, Magliano L, Brambilla L, Ugolini S, Pioli R. Development, reliability and acceptability of a new version of the DSM-IV Social and Occupational Functioning Assessment Scale (SOFAS) to assess routine social functioning. Acta Psychiatr Scand. 2000;101(4):323-329. doi:10.1111/j.1600-0447.2000.tb10933.x

43. Heinrichs DW, Hanlon TE, Carpenter WT Jr. The Quality of Life Scale: an instrument for rating the schizophrenic deficit syndrome. Schizophr Bull. 1984;10(3):388-398. doi:10.1093/schbul/10.3.388

44. Karow A, Moritz S, Lambert M, Schottle D, Naber D, EGOFORS Initiative. Remitted but still impaired? Symptomatic versus functional remission in patients with schizophrenia. Eur Psychiatry. 2012;27 (6):401-405. doi:10.1016/j.eurpsy.2011.01.012

45. Ascher-Svanum H, Novick D, Haro JM, Aguado J, Cui Z. Empirically driven definitions of "good," "moderate," and "poor" levels of functioning in the treatment of schizophrenia. Qual Life Res. 2013;22(8):2085-2094. doi:10.1007/s11136-012-0335-z

46. Tiryaki A, Ozkorumak E. Do the obsessive-compulsive symptoms have an effect in schizophrenia? Compr Psychiatry. 2010;51 (4):357-362. doi:10.1016/j.comppsych.2009.10.007 


\section{Publish your work in this journal}

Neuropsychiatric Disease and Treatment is an international, peerreviewed journal of clinical therapeutics and pharmacology focusing on concise rapid reporting of clinical or pre-clinical studies on a range of neuropsychiatric and neurological disorders. This journal is indexed on PubMed Central, the 'PsycINFO' database and CAS, and is the official journal of The International Neuropsychiatric Association (INA). The manuscript management system is completely online and includes a very quick and fair peer-review system, which is all easy to use. Visit http://www.dovepress.com/testimonials.php to read real quotes from published authors.

Submit your manuscript here: https://www.dovepress.com/neuropsychiatric-disease-and-treatment-journal 